\title{
Discrimination of dementia with Lewy bodies from Alzheimer's disease using voxel-based morphometry of white matter by statistical parametric mapping 8 plus diffeomorphic anatomic registration through exponentiated Lie algebra
}

\author{
Tomoya Nakatsuka • Etsuko Imabayashi • \\ Hiroshi Matsuda • Ryuji Sakakibara • Tsutomu Inaoka • \\ Hitoshi Terada
}

Received: 19 October 2012 / Accepted: 4 January 2013 /Published online: 16 January 2013

(C) The Author(s) 2013. This article is published with open access at Springerlink.com

\begin{abstract}
Introduction The purpose of this study was to identify brain atrophy specific for dementia with Lewy bodies (DLB) and to evaluate the discriminatory performance of this specific atrophy between DLB and Alzheimer's disease (AD).

Methods We retrospectively reviewed 60 DLB and 30 AD patients who had undergone 3D T1-weighted MRI. We randomly divided the DLB patients into two equal groups (A and B). First, we obtained a target volume of interest (VOI) for DLB-specific atrophy using correlation analysis of the percentage rate of significant whole white matter (WM) atrophy calculated using the Voxelbased Specific Regional Analysis System for Alzheimer's Disease (VSRAD) based on statistical parametric mapping 8 (SPM8) plus diffeomorphic anatomic
\end{abstract}

T. Nakatsuka $(\bowtie) \cdot$ T. Inaoka $\cdot H$. Terada

Department of Radiology, Toho University Sakura

Medical Center, 564-1, Shimoshizu,

Sakura, Chiba 285-8741, Japan

e-mail: gh23fhuw7@yahoo.co.jp

E. Imabayashi

Department of Nuclear Medicine, Saitama Medical

University International Medical Center, Hidaka,

Saitama, Japan

H. Matsuda

Integrative Brain Imaging Center, National Center

of Neurology and Psychiatry, Kodaira, Tokyo, Japan

R. Sakakibara

Division of Neurology, Department of Internal Medicine,

Toho University Sakura Medical Center,

Sakura, Chiba, Japan registration through exponentiated Lie algebra, with segmented WM images in group A. We then evaluated the usefulness of this target VOI for discriminating the remaining 30 DLB patients in group $B$ from the 30 AD patients. $Z$ score values in this target VOI obtained from VSRAD were used as the determinant in receiver operating characteristic (ROC) analysis.

Results Specific target VOIs for DLB were determined in the right-side dominant dorsal midbrain, right-side dominant dorsal pons, and bilateral cerebellum. ROC analysis revealed that the target VOI limited to the midbrain exhibited the highest area under the ROC curves of 0.75 . Conclusions DLB patients showed specific atrophy in the midbrain, pons, and cerebellum. Midbrain atrophy demonstrated the highest power for discriminating DLB and $\mathrm{AD}$. This approach may be useful for determining the contributions of DLB and AD pathologies to the dementia syndrome.

Keywords Dementia with Lewy bodies (DLB) - Alzheimer's disease (AD) - Statistical parametric mapping (SPM) - Voxelbased morphometry (VBM) · Voxel-based specific regional analysis system for Alzheimer's disease (VSRAD)

$\begin{array}{ll}\text { Abbreviations } \\ \text { AD } & \text { Alzheimer's disease } \\ \text { AUC } & \text { Areas under curve } \\ \text { DARTEL } & \begin{array}{l}\text { Diffeomorphic anatomical registration through } \\ \text { exponentiated Lie algebra }\end{array} \\ & \begin{array}{l}\text { Dementia with Lewy bodies } \\ \text { DLB }\end{array} \\ \text { GM } & \text { Gray matter } \\ \text { H/M } & \text { Heart-to-mediastinum }\end{array}$




$\begin{array}{ll}\text { MIBG } & \text { Meta-iodobenzylguanidine } \\ \text { MMSE } & \text { Mini-mental state examination } \\ \text { PDD } & \text { Parkinson's disease with dementia } \\ \text { PET } & \text { Positron emission tomography } \\ \text { ROC } & \text { Receiver operating characteristic } \\ \text { SD } & \text { Standard deviation } \\ \text { SPECT } & \text { Single photon emission computed tomography } \\ \text { SPM } & \text { Statistical parametric mapping } \\ \text { TE } & \text { Echo time } \\ \text { TI } & \text { Inversion time } \\ \text { TR } & \text { Repetition time } \\ \text { VBM } & \text { Voxel-based morphometry } \\ \text { VOI } & \text { Volume of interest } \\ \text { VSRAD } & \text { Voxel-based specific regional analysis system } \\ & \text { for Alzheimer's disease } \\ \text { WM } & \text { White matter }\end{array}$

\section{Introduction}

Dementia with Lewy bodies (DLB) is the second most common type of degenerative dementia, accounting for up to $30 \%$ of all cases of dementia [1]. In addition to dementia, visual hallucinations, fluctuating cognitive impairment, and parkinsonism are the main symptoms [2]. DLB is pathologically characterized by alpha-synuclein inclusions in the brainstem, subcortical nuclei, limbic, and neocortical areas [2]. Although the temporal sequence of symptoms and clinical features of DLB are considered to be different from those of Parkinson's disease with dementia (PDD), discriminating PDD and DLB is difficult in many cases. The accumulation of abnormal neuronal alpha-synuclein inclusions is the defining pathological process common to both PDD and DLB [3]. In this study, PDD and DLB are dealt with as a single pathogenic Lewy body disorder.

Nuclear medicine studies of the dopaminergic system are best suited for detecting pathological states in DLB. One of the more effective examinations for the differential diagnosis of DLB from Alzheimer's disease (AD) is ${ }^{123} \mathrm{I}-\mathrm{MIBG}$ myocardial scintigraphy. Yoshita et al. found that the delayed heart-to-mediastinum uptake $(\mathrm{H} / \mathrm{M})$ ratio had a sensitivity of $100 \%$, a specificity of $100 \%$, and a positive predictive value of $100 \%$ [4]. However, it should be noted that a decreased myocardial MIBG uptake is not specific of DLB; in fact, various heart diseases and diabetes may damage the postganglionic sympathetic neurons, leading to falsepositive MIBG findings [5]. Hypometabolism or hypoperfusion in the occipital cortex are also useful for distinguishing DLB from AD [6-8], although their accuracies are not so high. Commonly, MRI has been used to rule out other diseases manifesting dementia, such as cerebral infarction, chronic subdural hematoma, or normal pressure hydrocephalus, and this equipment is more widely used without radionuclides or radiation exposure, as is the case with positron emission tomography (PET) or single photon emission computed tomography (SPECT).

Voxel-based morphometry (VBM), which objectively assesses whole brain structure with voxel-by-voxel comparisons, has been developed to analyze tissue concentrations or volumes between subject groups to distinguish degenerative diseases with dementia [9]. Some studies comparing gray matter (GM) loss in DLB with that of $\mathrm{AD}$ by means of $\mathrm{VBM}$ have shown similar losses with relative preservation of the temporal lobes in DLB [10-12]. One study with a relatively large number of subjects found greater GM atrophy of the dorsal midbrain in DLB than in AD [12], while others have shown significant atrophy in specific subcortical regions, such as the putamen [13] and basal forebrain $[14,15]$.

Recently, the statistical parametric mapping 8 (SPM8) plus diffeomorphic anatomical registration through exponentiated Lie algebra (DARTEL; Wellcome Trust Centre for Neuroimaging, London, UK) method was developed for precise VBM [16] for both GM and white matter (WM). DARTEL was shown to improve registration and to provide precise, accurate localization of structural damage and functional overlays. However, Takahashi et al. showed no DLB-specific atrophy of WM compared to AD using SPM8 plus DARTEL [17]. The authors have insisted the GM attenuation in deep brain GM, including the dorsal midbrain, detected in previous studies may be attributed to incomplete registration to the template.

There have been only a few studies evaluating WM changes in DLB compared to AD and healthy controls with VBM [17, 18]. One conventional VBM study using SPM5 for analysis showed significant atrophy of $\mathrm{WM}$ in the pons and medulla oblongata in idiopathic Parkinson's disease compared to healthy controls [18]. The other study using SPM8 plus DARTEL for analysis showed no DLB-specific significant atrophy of WM compared to $\mathrm{AD}$ [17].

The purpose of our study was to identify a DLBspecific atrophy using SPM8 plus DARTEL and to evaluate the performance of this specific focal atrophy to discriminate between DLB and AD. Although the previous SPM8 plus DARTEL study did not find any DLB-specific atrophy [17], a recent study of antemortem MRI and postmortem neuropathologic classification of DLB and AD revealed that antemortem dorsal mesopontine atrophy was indicative of a high likelihood of DLB [19], leading us to take notice of brain stem 
atrophy in DLB. Because SPM8 plus DARTEL classifies most brain stem structures as white matter [16], we focused on white matter changes in the present study.

\section{Materials and methods}

We retrospectively reviewed 60 patients with DLB (32 men and 28 women, $77.3 \pm 5.8$ years of age) and 30 patients with $\mathrm{AD}(7$ men and 23 women, $76.7 \pm 5.7$ years of age), who had undergone 3D T1-weighted structural MRI from October 2009 to August 2011. All of the 60 patients with DLB had dementia and spontaneous features of parkinsonism. Mini-mental state examination (MMSE) scores were 20.8 \pm 4.1 (mean \pm SD). Some of them had fluctuating cognition with pronounced variations in attention and alertness, recurrent visual hallucinations that were typically well formed and detailed; thus, they were diagnosed as possible/probable DLB on the basis of the criteria proposed in the consortium on DLB international workshop [2]. All of the 60 patients with DLB revealed reduced $\mathrm{H} / \mathrm{M}$ ratios on delayed phase of ${ }^{123}$ I-MIBG myocardial scintigraphy which was used as an adjunct of differential diagnosis of DLB and AD. The 30 patients with AD were diagnosed as probable AD according to the National Institute of Neurologic and Communicative Disorders and Stroke and the Alzheimer's Disease and Related Disorders Association criteria $[20,21]$. The MMSE scores of the $30 \mathrm{AD}$ patients were $20.4 \pm 4$.1. All of $\mathrm{AD}$ patients did not reveal reduced $\mathrm{H} / \mathrm{M}$ ratios on either early or delayed phases of ${ }^{123}$ I-MIBG myocardial scintigraphy. The ethics committee of Toho University Sakura Medical Center approved this study and all subjects provided informed consent to participate. None of them had asymptomatic cerebral infarction detected by $\mathrm{T} 2$ weighted MRI.

All 90 patients underwent MRI examinations on a 1.5T Gyroscan (Philips, Best, the Netherlands). One hundred eighty 3D sections of a T1-weighted, magnetization-prepared rapid acquisition of gradientecho (MPRAGE) sequence were obtained in a sagittal orientation as $1-\mathrm{mm}$ thick gapless sections (FOV $240 \mathrm{~mm}$, TR $9.7 \mathrm{~ms}$, TE $4.6 \mathrm{~ms}$, flip angle $10^{\circ}$, and TI $525 \mathrm{~ms})$.

Using a free software program, the voxel-based specific regional analysis system for Alzheimer's disease (VSRAD) based on SPM8 plus DARTEL [22], we classified MRIs of all 90 patients into GM, WM, or cerebrospinal fluid images using a unified tissuesegmentation procedure after image-intensity nonuniformity correction, anatomically standardized to a customized template of WM, and then smoothed using an 8-mm full width at half maximum isotropic Gaussian kernel. This new VSRAD software was upgraded from the previous SPM2 version [23]. VSRAD provides statistical $Z$ score images for WM atrophy in each of the patients compared to that of the "normal" database of WM. The $Z$ score was defined as: ([control mean]-[individual value])/(control SD). The "normal" database bundled with VSRAD comprised 80 healthy volunteers ( 37 men and 43 women), aged 54 to 86 years who underwent 3D T1-weighted MPRAGE sequence using a $1.5 \mathrm{~T}$ Siemens Vision Plus scanner (Siemens, Erlangen, Germany). Their performance was within normal limits both on the Wechsler Memory Scale-Revised and Wechsler Adult Intelligence Scale-Revised [22]. Their MMSE scores ranged from 26 to $30 ; 29.1 \pm 1.2$.

We divided the 60 patients with DLB into two groups at random (group A: 30 patients, group B: 30 patients). We obtained extent of significant WM atrophy for the whole brain, that is, the percentage rate of the coordinates with a $Z$ value exceeding the threshold value of 2 in the whole brain, in each patient as compared with that of the "normal" database bundled with VSRAD in group A. Then, to detect target volumes of interest (VOI) for DLB correlation, we compared this whole brain percentage rate of WM atrophy with that of each voxel in the anatomically standardized and smoothed WM images in group A using SPM8 and their ages as a nuisance covariate. Global nuisance effects were accounted for by including the global covariate as a nuisance effect. The resulting set of values for each contrast of negative or positive correlation constituted statistical parametric maps of the $t$ statistic SPM $\{t\}$. Main effects used whole-brain analyses with an uncorrected threshold at voxel level of $p<0.001$ and a cluster false discovery rate for the multiple comparison correction.

We then evaluated the usefulness of this target VOI for discriminating the remaining 30 DLB patients in group B from the $30 \mathrm{AD}$ patients. VSRAD provided statistical $Z$ score images for WM atrophy in each of the patients compared to that of the "normal" database of WM. We obtained

Table 1 Regions in which WM negatively correlated with percentage of whole WM atrophy in 30 DLB patients with DLB (group A)

\begin{tabular}{llllll}
\hline Brain region & \multicolumn{3}{l}{ Talairach coordinates } & & \multirow{2}{*}{$t$ value } \\
\hline & Side & $x$ & $y$ & $z$ & \\
Cerebellum posterior lobe & $\mathrm{Rt}$ & 12 & -52 & -31 & 4.5 \\
Cerebellum anterior lobe & $\mathrm{Lt}$ & -12 & -42 & -21 & 3.9 \\
Midbrain & $\mathrm{Rt}$ & 4 & -24 & -11 & 3.4 \\
Pons & $\mathrm{Rt}$ & 4 & -39 & -33 & 3.2 \\
\hline
\end{tabular}


Fig. 1 Statistical parametric mapping (SPM) showing the negative correlation of the percentage of whole WM atrophy to that in various brain regions in the 30 DLB patients in group A
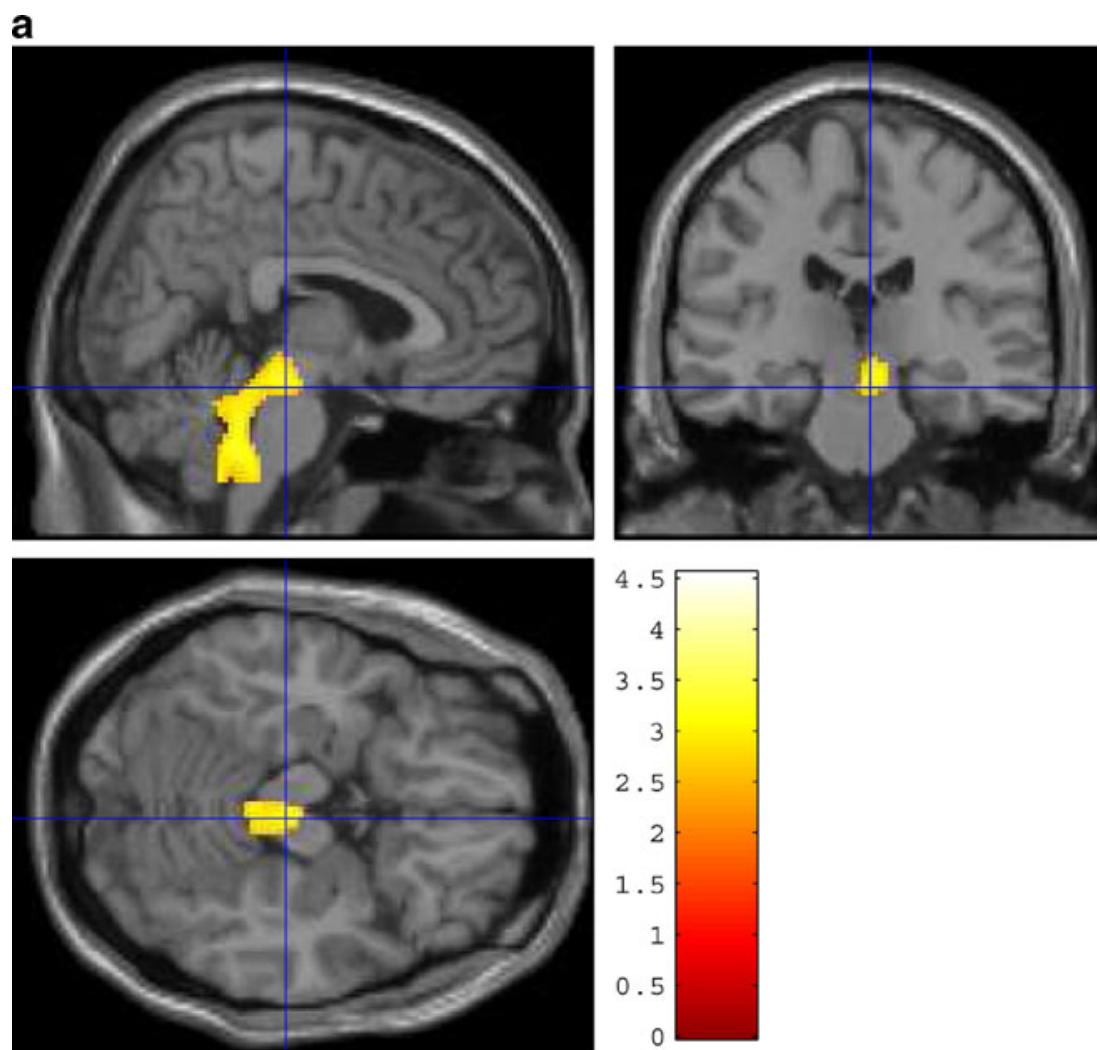

b


\section{$\operatorname{SPM}\left\{\mathrm{T}_{26}\right\}$}

the averaged positive $Z$ score in the target VOI with MRIcron (http://www.mccauslandcenter.sc.edu/mricro/mricron/). Using these averaged positive $Z$ scores in the target VOI as a threshold, we used JMP 7.0 (SAS Institute, Cary, North Carolina) to determine receiver operating characteristic (ROC) curves for discriminating DLB and $\mathrm{AD}$ patients. The program 
calculated the areas under the ROC curves (AUC; probability of concordance), sensitivity, specificity, and accuracy.

\section{Results}

DLB patients in group A exhibited a significant negative correlation of their percentage rate of significant whole WM atrophy with that of voxels in the right-side dominant dorsal midbrain, right-side dominant dorsal pons, and bilateral cerebellum and no significant positive correlations (Table 1, Fig. 1). The mean percentage rate of significant whole WM atrophy was $5.3 \pm 7.7 \%(0-35.6)$. From this negative correlation, we determined a target VOI for DLB-specific atrophy. The target VOI was divided into three parts, and we created five target VOIs, consisting of the dorsal midbrain, dorsal pons, cerebellum, midbrain plus pons, and midbrain plus pons plus cerebellum (Fig. 2). ROC analysis using the averaged positive $Z$ scores in these five target VOIs was performed to discriminate the DLB patients in group B from the AD patients (Fig. 3). A target VOI limited to the midbrain exhibited the highest AUC of 0.75 , sensitivity of $80 \%$, specificity of $64 \%$, and accuracy of $72 \%$. A target VOI limited to the midbrain plus pons exhibited an AUC of 0.74 , sensitivity of $60 \%$, specificity $90 \%$, and accuracy of $75 \%$. A target VOI limited to the pons exhibited an AUC of 0.68 , sensitivity of $47 \%$, specificity of $93 \%$, and accuracy of $70 \%$. A target VOI limited to the cerebellum exhibited an AUC of 0.50 , sensitivity of $20 \%$, specificity of $90 \%$, and accuracy of $55 \%$. A target VOI encompassing the midbrain plus pons and cerebellum exhibited an AUC of 0.55 , sensitivity of $23 \%$, specificity of $90 \%$, and accuracy of $56.5 \%$.
Fig. 2 Target volumes of interest (VOIs) for DLBspecific atrophy: a midbrain, b pons, $\mathbf{c}$ midbrain plus pons, $\mathbf{d}$ cerebellum, and e midbrain plus pons plus cerebellum
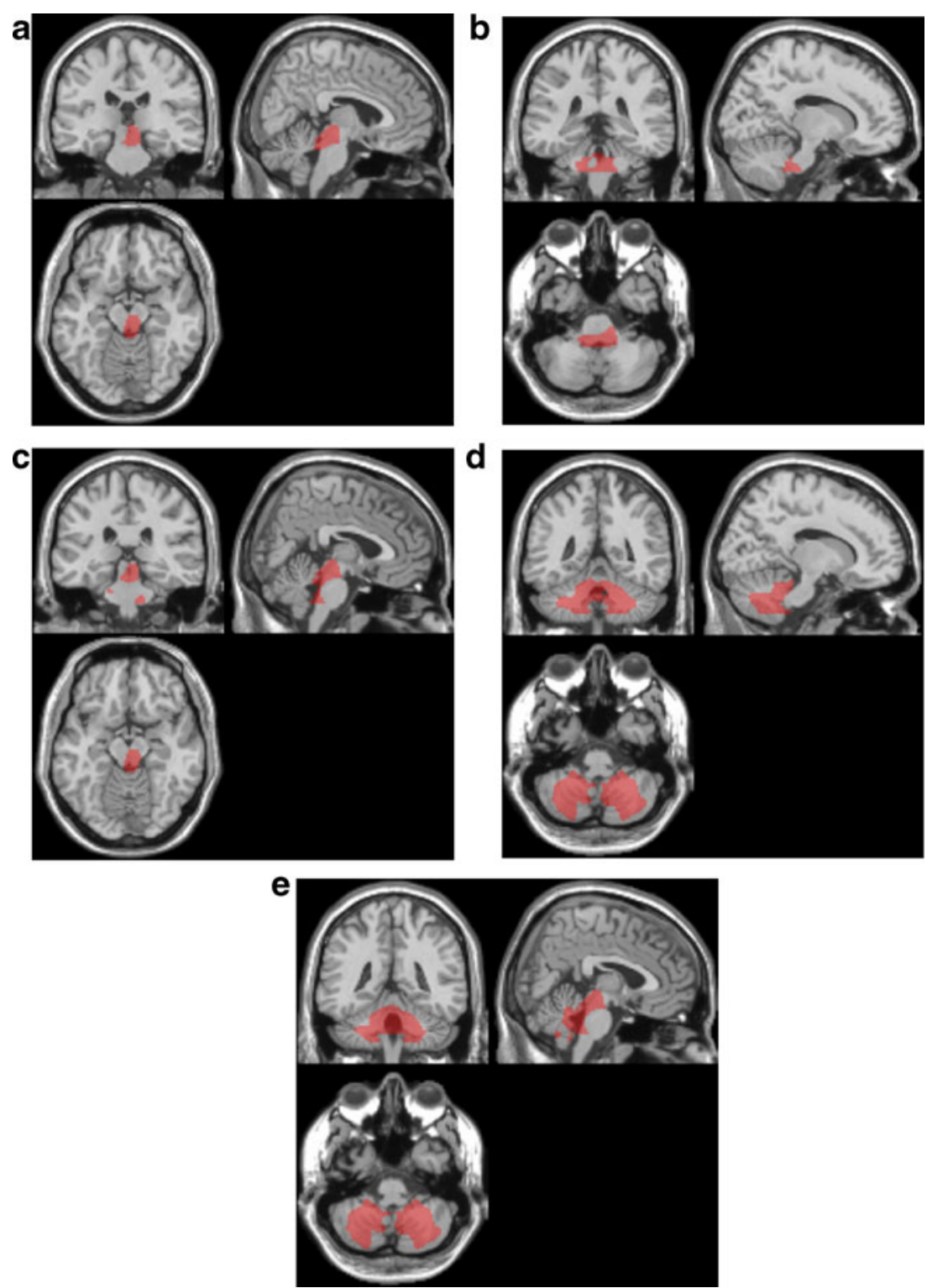
Fig. 3 ROC curves for discriminating DLB and AD patients using averaged positive $Z$ scores in target VOIs as a threshold: a midbrain, $\mathbf{b}$ pons, $\mathbf{c}$ midbrain plus pons, $\mathbf{d}$ cerebellum, and e midbrain plus pons plus cerebellum


d

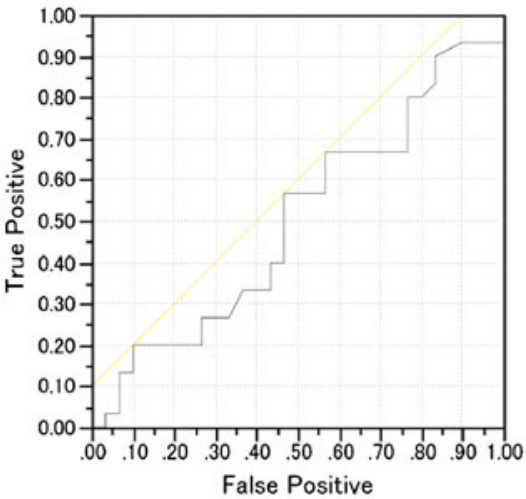

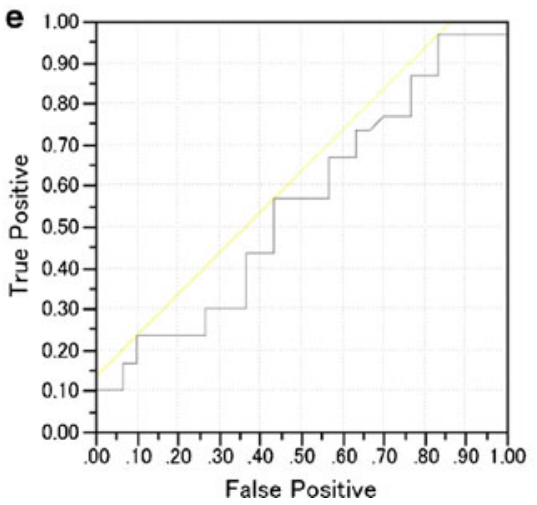

\section{Discussion}

The present study demonstrated DLB-specific WM atrophy in the right-side dominant dorsal midbrain, right-side dominant dorsal pons, and bilateral cerebellum. Right-side dominancy may result from the feature of the present cohort of DLB patients. The WM atrophy in these areas was more frequently observed in DLB than in AD. Of these areas, midbrain atrophy exhibited the most powerful discrimination of DLB and AD. These findings are consistent with pathological findings showing that Lewy bodies move up the brainstem into the midbrain and then to the forebrain before spreading into the cortex [18, 24, 25].

Takahashi et al. found no significant DLB-specific WM and GM atrophy compared to AD using SPM8 plus DARTEL
[17]. Both our current study and theirs accepted an uncorrected threshold of $p<0.001$, but only ours used more stringent analysis of a cluster false discovery rate of $p<0.05$ for the multiple comparison correction. This inconsistency might arise from the differences in the cluster analyses or variability in the clinical cohorts (e.g., dementia severity, symptom presentation) of DLB and AD, although age and MMSE scores did not differ significantly in the two studies.

Distinguishing DLB from AD is important for choosing appropriate treatments, because clinical symptoms are sometimes too similar to distinguish between the two. Our ROC results showed that the use of VBM with SPM8 plus DARTEL may not be as accurate as using ${ }^{123}$ I-MIBG myocardial scintigraphy [4]. This may be partly due to the fact that a majority of DLB patients have overlapping AD and DLB 
pathologies. Thus, brain stem atrophy revealed by MRI may play a complementary role in determining the contribution of DLB and AD pathologies to the dementia syndrome.

Our study was possibly limited by several factors. Since we did not have MRI data of normal control group, we searched DLB-specific area for WM atrophy using a linear correlation analysis of each patient's WM image with significant atrophy rate for the whole brain WM as compared with "normal" database bundled with VSRAD. This indirect comparison between different MRI scanners should be validated in a further study based on the on-site normal database. Whether the present target VOI obtained from DLB versus normal is the best for DLB versus $\mathrm{AD}$ discrimination should be also further evaluated in direct comparison of a larger number of DLB and AD patients. However the present target VOI locates in the midbrain where DLB showed significant atrophy compared to $\mathrm{AD}$ in previous studies $[12$, 19]. We enrolled patients not only with probable DLB, but also those with possible DLB, even though their ${ }^{123}$ I MIBGSPECT results showed reduced $\mathrm{H} / \mathrm{M}$ ratios on delayed phase images, and we did not assess the clinical severities (i.e., Hoehn and Yahr Scores) of the DLB patients. In the future, we intend to evaluate the usefulness of this target VOI for differentiating other dementia diseases using VBM with SPM8 plus DARTEL as well as the usefulness of combining this VBM analysis with other imaging modalities, including SPECT and PET and their relationship to the findings of postmortem neuropathology.

\section{Conclusions}

Our VBM analysis using SPM8 plus DARTEL demonstrated significant WM atrophy in the dorsal midbrain, dorsal pons, and cerebellum in the patients with DLB. Of these areas with DLB-specific WM atrophy, the midbrain atrophy exhibited the highest power for discriminating DLB from AD. This VBM approach may be useful for determining the contributions of DLB and AD pathologies to the dementia syndrome.

Conflict of interest We declare that we have no conflict of interest.

Open Access This article is distributed under the terms of the Creative Commons Attribution License which permits any use, distribution, and reproduction in any medium, provided the original author(s) and the source are credited.

\section{References}

1. Zaccai J, McCracken C, Brayne C (2005) A systematic review of prevalence and incidence studies of dementia with Lewy bodies. Age Ageing 34:561-566
2. McKeith IG, Dickson DW, Lowe J et al (2005) Diagnosis and management of dementia with Lewy bodies: third report of the DLB Consortium. Neurology 65:1863-1872

3. Lippa CF, Duda JE, Grossman M et al (2007) DLB and PDD boundary issues: diagnosis, treatment, molecular pathology, and biomarkers. Neurology 68:812-819

4. Yoshita M, Taki J, Yokoyama K, Noguchi-Shinohara M, Matsumoto Y, Nakajima K, Yamada M (2006) Value of ${ }^{123}$ I-MIBG radioactivity in the differential diagnosis of DLB from AD. Neurology 66:1850-1854

5. Treglia G, Cason E, Stefanelli A, Cocciolillo F, Di Giuda D, Fagioli G, Giordano A (2012) MIBG scintigraphy in differential diagnosis of Parkinsonism: a meta-analysis. Clin Auton Res 22:43-55

6. Small GW (2004) Neuroimaging as a diagnostic tool in dementia with Lewy bodies. Dement Geriatr Cogn Disord 17(suppl 1):2531

7. Ishii K, Soma T, Kono AK, Sofue K, Miyamoto N, Yoshikawa T, Mori E, Murase K (2007) Comparison of regional brain volume and glucose metabolism between patients with mild dementia with Lewy bodies and those with mild Alzheimer's disease. J Nucl Med 48:704-711

8. Kono AK, Ishii K, Sofue K, Miyamoto N, Sakamoto S, Mori E (2007) Fully automatic differential diagnosis system for dementia with Lewy bodies and Alzheimer's disease using FDG-PET and 3D-SSP. Eur J Nucl Med Mol Imaging 34:1490-1497

9. Ashburner J, Friston KJ (2000) Voxel-based morphometry-the methods. NeuroImage 11(6 Pt1):805-821

10. Burton EJ, Karas G, Paling SM, Barber R, Williams ED, Ballard CG, McKeith IG, Scheltens P, Barkhof F, O'Brien JT (2002) Patterns of cerebral atrophy in dementia with Lewy bodies using voxel-based morphometry. NeuroImage 17:618-630

11. Burton EJ, McKeith IG, Burn DJ, Williams ED, O’Brien JT (2004) Cerebral atrophy in Parkinson's disease with and without dementia: a comparison with Alzheimer's disease, dementia with Lewy bodies and controls. Brain 127(Pt 4):791-800

12. Whitwell JL, Weigand SD, Shiung MM et al (2007) Focal atrophy in dementia with Lewy bodies on MRI: a distinct pattern from Alzheimer's disease. Brain 130(Pt 3):708-719

13. Cousins DA, Burton EJ, Burn D, Gholkar A, McKeith IG, O'Brien JT (2003) Atrophy of the putamen in dementia with Lewy bodies but not Alzheimer's disease: an MRI study. Neurology 61:1191-1195

14. Brenneis C, Wenning GK, Egger KE et al (2004) Basal forebrain atrophy is a distinctive pattern in dementia with Lewy bodies. NeuroReport 15:1711-1714

15. Hanyu H, Shimizu S, Tanaka Y, Hirao K, Iwamoto T, Abe K (2006) MR features of the substantia innominate and therapeutic implications in dementias. Neurobiol Aging 28:548-554

16. Ashburner J (2007) A fast diffeomorphic image registration algorithm. NeuroImage 38:95-113

17. Takahashi R, Ishii K, Miyamoto N, Yoshikawa T, Shimada K, Ohkawa S, Kakigi T, Yokoyama K (2010) Measurement of gray and white matter atrophy in dementia with Lewy bodies using diffeomorphic anatomic registration through exponentiated lie algebra: a comparison with conventional voxel-based morphometry. AJNR Am J Neuroradiol 31:1873-1878

18. Jubault T, Brambati SM, Degroot C, Kullmann B, Strafella AP, Lafontaine AL, Chouinard S, Monchi O (2009) Regional brain stem atrophy in idiopathic Parkinson's disease detected by anatomical MRI. PLoS One 4:e8247

19. Kantarci K, Ferman TJ, Boeve BF et al (2012) Focal atrophy on MRI and neuropathologic classification of dementia with Lewy bodies. Neurology 79:553-560

20. McKhann G, Drachman D, Folstein M, Katzman R, Price D, Stadlan EM (1984) Clinical diagnosis of Alzheimer's disease: report of the NINCDS-ADRDA work group under the auspices 
of Department of Health and Human Services taskforce on Alzheimer's disease. Neurology 34:939-944

21. Jack CR Jr, Albert MS, Knopman DS, McKhann GM, Sperling RA, Carrillo MC, Thies B, Phelps CH (2011) Introduction to the recommendations from the National Institute on AgingAlzheimer's Association workgroups on diagnostic guidelines for Alzheimer's disease. Alzheimers Dement 7:257-262

22. Matsuda H, Mizumura S, Nemoto K, Yamashita F, Imabayashi E, Sato N, Asada T (2012) Automatic voxel-based morphometry of structural MRI by SPM8 plus diffeomorphic anatomic registration through exponentiated Lie algebra improves the diagnosis of probable Alzheimer disease. AJNR Am J Neuroradiol 33:11091114

23. Hirata Y, Matsuda H, Nemoto K, Ohnishi T, Hirao K, Yamashita F, Asada T, Iwabuchi S, Samejima H (2005) Voxel-based morphometry to discriminate early Alzheimer's disease from controls. Neurosci Lett 382:269-274

24. Braak H, Ghebremedhin E, Rub U, Bratzke H, Del Tredici K (2004) Stages in the development of Parkinson's disease-related pathology. Cell Tissue Res 318:121-134

25. Jellinger KA (2004) Lewy body-related alpha-synucleinopathy in the aged human brain. J Neural Transm 111:1219-1235 